\title{
A flow-cytometric study of the effect of myeloperoxidase on stallion spermatozoal motility and structure
}
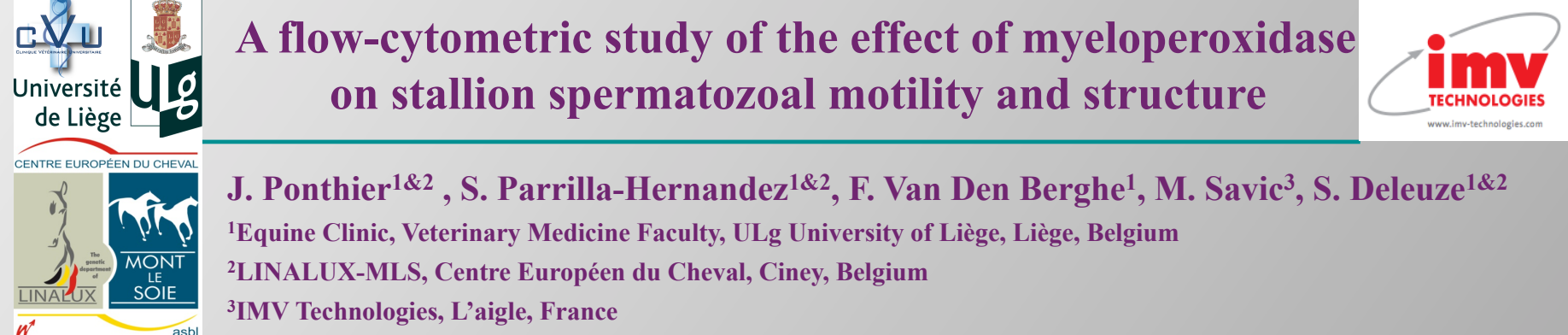

J. Ponthier ${ }^{1 \& 2}$, S. Parrilla-Hernandez ${ }^{1 \& 2}$, F. Van Den Berghe ${ }^{1}$, M. Savic ${ }^{3}$, S. Deleuze ${ }^{1 \& 2}$

${ }^{1}$ Equine Clinic, Veterinary Medicine Faculty, ULg University of Liège, Liège, Belgium

${ }^{2}$ LINALUX-MLS, Centre Européen du Cheval, Ciney, Belgium

${ }^{3}$ IMV Technologies, L'aigle, France

\section{Introduction}

\section{Myeloperoxidase (MPO):}

$=$ Pro-oxidant enzyme contained in and released by neutrophils

- Increased MPO concentrations observed in poor post-thaw quality semen in the equine ${ }^{1}$

Aim of this study was to observe effect of active MPO addition in fresh purified semen on:

- Semen motility

- Membrane and acrosome integrity

- Mitochondrial potential and apoptosis induction

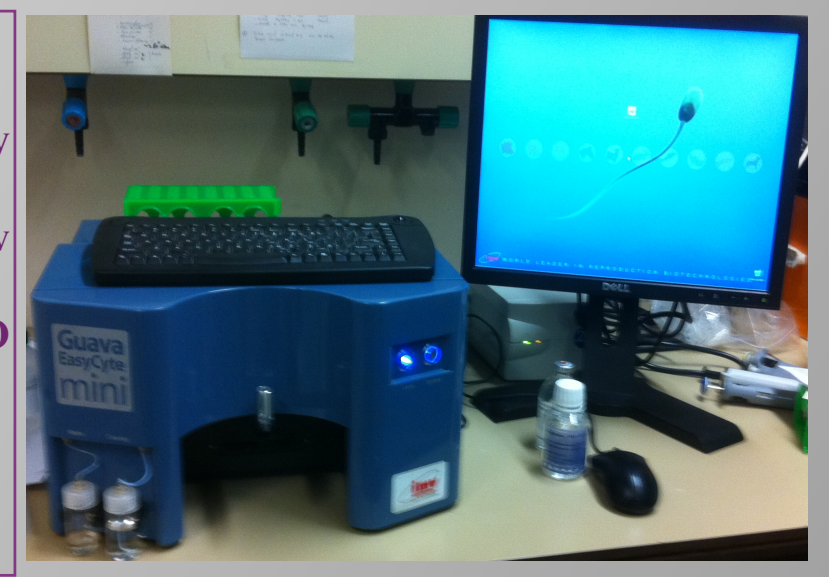

Material and methods

Animals: 1 Pony stallions and 2 draft stallions

Experimental design:

Semen collected 4 times

- Cushioned centrifugation in INRA96 $6^{\circledR}$

- Density Gradient centrifugation ${ }^{2}$ (Bottom Layer, Nidacon ${ }^{\circledR}$ )

- Pellet rediluted $\left(100 \times 10^{6} \mathrm{spz} / \mathrm{ml}\right)$ in and incubated $(2 \mathrm{~h})$ :

- Pure INRA96 ${ }^{\circledR}$ (Blank)

- $5 \mathrm{ng} / \mathrm{ml}$ MPO solution in INRA96 ${ }^{\circledR}$

- $50 \mathrm{ng} / \mathrm{ml}$ MPO solution in INRA96 ${ }^{\circledR}$

Semen analysis:

Motility: CASA analysis

Flow cytometry: IMV Guava Easycyte Mini ${ }^{\circledR}$

-Membrane and acrosome integrity: PI \& PNA

-Mitochondrial potential and apoptosis: JC-1 \& 7-AAD

Statistical methods:

-Kruskal-Wallis test was used to compare parameters obtained.

Statistical significance established at $\mathrm{p}<0.05$

\section{Results}

-No stallion effect observed after density gradient centrifugation

-Progressive Motility decreased by both MPO concentrations $(\mathrm{p}=0.001)$

-No effect on Total Motility

- No effect of purified MPO addition in semen on:

-Membrane and acrosome integrity

-Mitochondrial potential

-Apoptosis induction

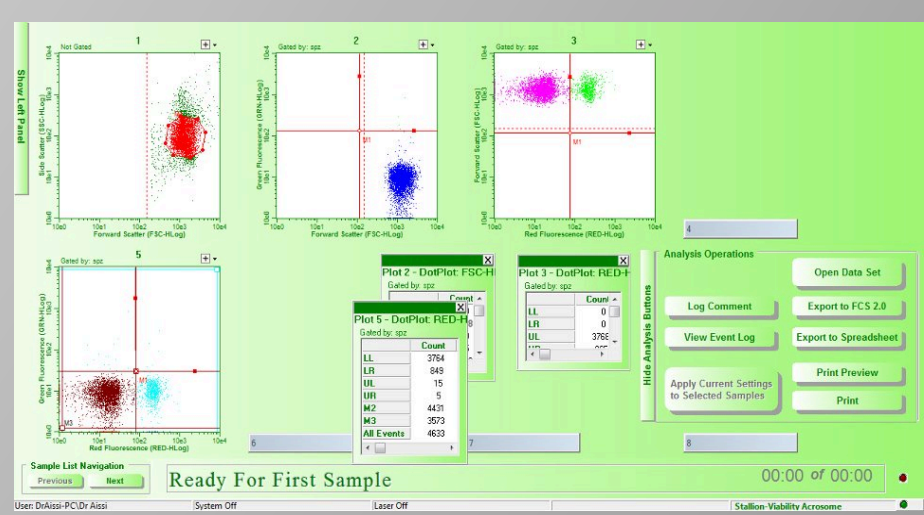

Discussion

- Results observed with MPO addition in semen are similar to previous results using xanthine oxidase ROS production system ${ }^{3}$ :

-Decrease of progressive motility;

-No effect on membrane and acrosome intergity;

-No effect on mitochondrial potential or apoptosis.

-Effect of MPO and ROS on motility was not explained by lesions of membrane, acrosome or mitochondria.

- Further studies should determine if motility decrease due to MPO is not associated to lipoperoxidation, as previously observed with xanthine oxidase ${ }^{3}$.

Conclusions

-MPO addition induces progressive motility decrease without affecting mitochondrial potential, membrane and acrosome integrity

-Further studies should focus on other lesions induced by MPO on spermatozoa 